\title{
IRRADIAÇÃO DE ARROZ E ALTERAÇÕES NAS SUAS PROPRIEDADES FÍSICO-QUÍMICAS E SENSORIAIS
}

\author{
LUÍS FERNANDO POLESI* \\ JULIANA PIEDADE* \\ MARIA JULIA DE MIGUEL AMISTÁ** \\ REGINA C. R. MIRANDA MILAGRES* \\ MARTA HELENA FILLETT SPOTO*** \\ SILENE BRUDER SILVEIRA SARMENTO**** \\ SOLANGE GUIDOLIN CANNIATTI-BRAZACA*****
}

\begin{abstract}
O objetivo do presente trabalho foi avaliar as propriedades físicoquímicas e a aceitação sensorial do arroz submetido à radiação gama. As amostras de arroz comercial (IRGA417), exceto o controle, foram submetidas às doses de radiação gama de 1,2 e $5 \mathrm{kGy}$, na taxa de dose de $0,5 \mathrm{kGy} / \mathrm{h}$. A irradiação promoveu redução no teor de amido e amarelecimento dos grãos. Quanto às propriedades de cocção, o arroz apresentou menor absorção de água e volume de expansão e maior perda de sólidos na água de cocção, conforme o aumento nas doses de radiação. A análise sensorial revelou boa aceitabilidade do arroz pelos julgadores, exceto para a dose de 5 kGy. A dose de 1 kGy destacou-se como a melhor para irradiação de arroz, pois não apresentou diferença do controle em nenhum parâmetro estudado.
\end{abstract}

PALAVRAS-CHAVE: RADIAÇÃO GAMA; Oryza sativa; COMPOSIÇÃO CENTESIMAL; AMIDO; PROPRIEDADES DE COCÇÃO.

* Doutorandos, Programa de Pós-graduação em Ciências, Centro de Energia Nuclear na Agricultura/ Universidade de São Paulo (CENA/USP), Piracicaba, SP (e-mail: Ifpolesi@yahoo.com.br; jpiedade@ cena.usp.br; rmilagres@cena.usp.br;).

** Mestranda em Ciência e Tecnologia de Alimentos, Escola Superior de Agricultura Luiz de Queirozl Universidade de São Paulo (ESALQ/USP), Piracicaba, SP (e-mail: amistamjm@gmail.com).

*** Doutora em Tecnologia Nuclear, ESALQ/USP, Piracicaba, SP (e-mail: mhfspoto@esalq.usp.br).

**** Doutora em Ciência dos Alimentos, ESALQ/USP, Piracicaba, SP (e-mail: sbssarme@usp.br).

***** Doutora em Ciência dos Alimentos, ESALQ/USP, Piracicaba, SP (e-mail: sgcbraza@usp.br). 


\section{INTRODUÇÃO}

O arroz (Oryza sativa L.), um dos cereais mais produzidos e consumidos no mundo, caracteriza-se como o principal alimento para mais da metade da população mundial (WALTER, MARCHEZAN e AVILA, 2008). De acordo com a Pesquisa de Orçamentos Familiares, realizada no Brasil entre 2008 e 2009, esse cereal alcançou a segunda maior média de consumo diário "per capita" (160,3 g/dia). O arroz faz parte da cultura brasileira, sendo apreciado por todos os estratos sociais e faixas etárias (IBGE, 2011; FERREIRA et al., 2005).

$\mathrm{O}$ arroz constitui importante fonte de energia na alimentação humana, devido à alta concentração de amido, além de fornecer proteínas, vitaminas e minerais (WALTER, MARCHEZAN e AVILA, 2008). Uma das maneiras de se preservar a integridade física do grão de arroz, mantendo suas características nutricionais, consiste no emprego da radiação gama, amplamente utilizada para proteger os alimentos da infestação de insetos e da contaminação microbiológica durante o armazenamento. Entretanto, a radiação gama pode modificar as propriedades químicas e físicas do amido (BAO, AO e JANE, 2005). Seu efeito na composição química dos produtos alimentícios tem recebido ampla atenção devido às alterações provocadas que podem ser desejáveis ou não. Além disso, podem existir perdas de ordem sensorial, como cor, sabor, textura e odor, passíveis de ocorrer em qualquer processo de conservação (FARKAS, 2006).

As propriedades sensoriais são os atributos dos alimentos detectados por meio dos sentidos, como visão, olfato, paladar e audição. A aparência geral, a textura e a cor são propriedades sensoriais muito importantes dos alimentos, sendo mais fáceis de avaliar do que o sabor, fenômeno complexo e dinâmico, relacionado principalmente ao olfato e ao gosto. O olfato compõe a fisiologia do sabor, pois o aroma é percebido antes do gosto (LOZANO, 1999).

Dentre os testes sensoriais, os afetivos ou hedônicos têm como proposta inicial a obtenção de respostas de preferência ou aceitação para determinado produto avaliado ou característica específica. Também permitem avaliar atitudes subjetivas de forma individual ou em relação a outros indivíduos. São utilizados quando se necessita conhecer o "status afetivo" dos consumidores em relação ao produto e para isso são utilizadas escalas hedônicas previamente estabelecidas, que variam gradativamente com base nos atributos gosto e desgosto (CHAVES e SPROESSER, 1996).

Como a irradiação pode alterar os componentes presentes no arroz, o objetivo deste trabalho foi avaliar as propriedades físico-químicas e a aceitação sensorial do arroz submetido à radiação gama.

\section{MATERIAL E MÉTODOS}

Foram utilizados grãos de arroz da cultivar IRGA 417, comercialmente disponíveis, e reagentes de grau analítico nas análises físico-químicas.

\subsection{COMPOSIÇÃO CENTESIMAL}

Analisou-se a composição centesimal dos grãos de arroz cru somente na amostra não tratada por radiação gama. Os teores de umidade, cinzas, proteínas e lipídeos foram analisados segundo os métodos da AOAC (2005), sendo o teor de carboidratos totais estimado por diferença.

\subsection{TRATAMENTO DE IRRADIAÇÃO}

Os grãos de arroz cru, exceto o controle, foram submetidos às doses de radiação gama (comumente utilizadas para cereais) de 1, 2 e $5 \mathrm{kGy}$, na taxa de 0,5 kGy/h, em irradiador Multipropósito de ${ }^{60} \mathrm{Co}$ em temperatura ambiente.

\subsection{PROPRIEDADES FÍSICO-QUÍMICAS}

As amostras de arroz cru foram moídas, peneiradas $(250 \mu \mathrm{m})$ e avaliadas quanto ao teor de amido total pelo método de Rickard e Behn (1987) e teor de açúcares redutores pelo método de Somogy-Nelson (SOMOGY, 1945). 
As propriedades de cocção do arroz foram avaliadas pelos testes de absorção de água (AA), volume de expansão (VE), perda de sólidos (PS) e turbidez da água de cocção (T). Determinou-se a AA em $1 \mathrm{~g}$ de arroz cru, adicionado de $15 \mathrm{~mL}$ de água destilada em tubo de ensaio vedado, por 25 min em banho de água fervente. Efetuou-se o cálculo conforme a expressão: $A A=$ (peso arroz cozido/peso do arroz cru) ${ }^{*} 100$ (BASSINELLO, ROCHA e COBUCCI, 2004). Determinou-se a PS pela secagem da água de cocção do arroz a $105^{\circ} \mathrm{C}$ durante 12 horas, usando-se a expressão: PS = (peso do resíduo de evaporação/peso arroz cru)*100 (CUI et al., 2010). Mediu-se o VE para o arroz cru e após cocção, utilizando o método de deslocamento de acordo com Cui et al. (2010), calculado como: $\mathrm{VE}=$ (volume do arroz cozido/volume do arroz cru) ${ }^{*} 100$. A T foi medida pela transmitância a $650 \mathrm{~nm}$ em espectrofotômetro, utilizando-se água destilada como referência e valor de transmitância de 100 (ONG e BLANSHARD, 1995).

Avaliou-se a cor instrumental dos grãos de arroz cru e cozido utilizando colorímetro Minolta, Modelo Chroma Meter CR-400. Os resultados foram expressos na escala CIELCh, em que a componente axial L é conhecida como luminosidade e se estende de 0 (preto) a 100 (branco), o ângulo de tom ou "Hue" $\left(0^{\circ} \leq \mathrm{h}^{*} \leq 360^{\circ}\right)$, que varia na direção angular e representa as diferentes cores existentes, e o croma que varia na direção radial e representa a pureza de determinada cor em relação ao cinza (MINOLTA, 2007).

\subsection{ANÁLISES MICROBIOLÓGICAS}

A qualidade microbiológica das amostras do arroz cozido submetido à análise sensorial foi analisada quanto à sua microbiota contaminante de acordo com o preconizado pela RDC $\mathrm{n}^{\circ} 12$ (BRASIL, 2001). Foram realizados testes para coliformes totais e termotolerantes, Bacillus cereus e Estafilococos coagulase positiva, conforme os procedimentos propostos pelo Compendium of methods for the microbiological examination of foods (APHA, 2001), e Salmonella sp. de acordo com o método 17.9.33 da AOAC (2005).

\subsection{ANÁLISE SENSORIAL}

As amostras foram cozidas para a análise sensorial, utilizando-se $50 \mathrm{~g}$ de arroz, $100 \mathrm{~mL}$ de água destilada, $2 \mathrm{~mL}$ de óleo de soja e $0,5 \mathrm{~g}$ de $\mathrm{NaCl}$, com cozimento a vapor em placa de petri de $15 \mathrm{~cm}$ por $30 \mathrm{~min}$ (BASSINELLO, ROCHA e COBUCCI, 2004). A realização do teste foi previamente aprovada pelo Comitê de Ética em Pesquisa, da Escola Superior de Agricultura Luiz de Queiroz da Universidade de São Paulo, sob o Protocolo n 52/2009.

Realizou-se o teste de aceitação sensorial das amostras no laboratório de análise sensorial quanto à aparência, aroma, sabor, textura e cor, utilizando-se escala hedônica de nove pontos ( 1 = desgostei muitíssimo, 9 = gostei muitíssimo). Participaram do teste 40 julgadores não treinados, adultos (entre 18 a 50 anos) de ambos os sexos e consumidores de arroz. A avaliação sensorial foi realizada em cabines individuais, com as amostras apresentadas aos julgadores na temperatura usual de consumo (aproximadamente $50^{\circ} \mathrm{C}$ ), em recipientes codificados com algarismos de três dígitos, juntamente com a ficha de avaliação (DUTCOSKY, 1996).

\subsection{ANÁLISE ESTATÍSTICA}

Adotou-se delineamento experimental inteiramente casualizado. Os resultados foram submetidos à análise de variância (ANOVA) e ao Teste de Tukey $(p<0,05)$ para comparação de médias utilizando-se o sistema estatístico SAS, versão 9.1 (SAS, 2002/2003). A correlação de Pearson entre as variáveis, cor instrumental e cor sensorial foi estimada pelo SAS.

\section{RESULTADOS E DISCUSSÃO}

\subsection{COMPOSIÇÃO CENTESIMAL}

Analisou-se a composição centesimal apenas do arroz controle (não irradiado), pois a 
radiação gama não altera de maneira significativa a composição centesimal, conforme os estudos realizados por Zanão et al. (2009).

A umidade do arroz $(11,29 \% \pm 0,04)$ ficou abaixo do limite máximo de $14 \%$, preconizado pela legislação vigente (BRASIL, 2009), o que auxilia a conservação do produto.

Os teores de cinzas $(0,34 \% \pm 0,01)$ e proteínas $(6,72 \% \pm 0,10)$ mostraram-se de acordo com os valores apresentados na literatura (USP, 2011; ZANÃO et al., 2009), entre 0,3-0,5 e 6,7$8,8 \%$, respectivamente.

O teor de lipídeos $(0,23 \% \pm 0,02)$ ficou abaixo dos valores encontrados em outros trabalhos, que reportam entre 0,3 e 0,9\% (UNICAMP, 2006; USP, 2011; ZANÃO et al., 2009), enquanto os carboidratos (81,42\%) apresentaram-se acima, 78,8-79,9\% (UNICAMP, 2006; USP, 2011; ZANÃO et al., 2009). Essas variações ocorrem, principalmente, devido diferença na cultivar de arroz.

\subsection{PROPRIEDADES FÍSICO-QUÍMICAS}

O teor de amido do arroz (Tabela 1) não foi alterado na dose de radiação de $1 \mathrm{kGy}$, sendo reduzido nas doses de $2 \mathrm{kGy}$ e $5 \mathrm{kGy}$. Essa redução pode ser devida à diminuição da capacidade de atuação das enzimas digestivas no decurso da análise, pois durante a irradiação podem se formar ligações cruzadas, ligações $\beta$ e grupos carboxilas, que dificultam a digestibilidade do amido (BHAT e KARIM, 2009; CHUNG e LIU 2009). Já o teor de açúcares redutores aumentou para a dose de $2 \mathrm{kGy}$, mas não diferiu $(p<0,05)$ do controle nas doses de 1 kGy e $5 \mathrm{kG}$. A aplicação de radiação ionizante gera radicais livres que são capazes de induzir alterações moleculares e fragmentação do amido. Durante o tratamento de radiação gama, as ligações glicosídicas são quebradas nos grânulos de amido ocasionando a decomposição das macromoléculas e a criação de macromoléculas com cadeias menores. No entanto, pode ocorrer a formação de ligações cruzadas entre as cadeias do amido, com aumento da massa molecular (BHAT e KARIM, 2009).

TABELA 1 - TEORES DE AMIDO E AÇÚCARES REDUTORES (\% BASE SECA)

\begin{tabular}{ccccc}
\hline \multirow{2}{*}{ COMPONENTES } & Controle & Dose $1 \mathrm{kGy}$ & Dose 2 kGy & Dose $5 \mathrm{kGy}$ \\
\cline { 2 - 4 } & $91,34 \pm 0,49^{\mathrm{a}}$ & $91,66 \pm 0,77^{\mathrm{a}}$ & $84,06 \pm 1,16^{\mathrm{b}}$ & $77,59 \pm 0,72^{\mathrm{c}}$ \\
\hline Amido (\%) & $0,74 \pm 0,04^{\mathrm{b}}$ & $0,73 \pm 0,11^{\mathrm{b}}$ & $1,06 \pm 0,09^{\mathrm{a}}$ & $0,94 \pm 0,07^{\mathrm{ab}}$ \\
\hline
\end{tabular}

Valores reportados como média \pm desvio padrão. Médias seguidas pela mesma letra na linha não diferem entre si pelo Teste de Tukey $(p<0,05)$.

Com relação às propriedades de cocção, as amostras apresentaram redução na absorção de água e no volume de expansão do arroz cozido com o aumento da dose de radiação aplicada (Tabela 2). No entanto, a dose de $1 \mathrm{kG}$ yão mostrou diferença significativa $(p<0,05)$ em relação ao controle. Os grãos de arroz com doses de radiação mais elevadas absorveram menos água e se expandiram menos, promovendo rendimento final menor. Wootton, Djojonegoro e Driscoll (1988) também observaram redução na absorção de água em arroz irradiado com o aumento das doses de radiação.

A perda de sólidos na água de cocção (Tabela 2) foi crescente com as doses aplicadas, no entanto, apenas a maior dose diferiu $(p<0,05)$ do controle. Como a irradiação promove a quebra das moléculas no grão de arroz ocorre aumento de moléculas menores e mais fáceis de serem lixiviadas. Desse modo, os sólidos que são levados para fora do grão aumentam durante a cocção. Elevação na perda de sólidos em arroz irradiado foi reportada na literatura e atribuída à degradação da estrutura do grão de arroz que permite a liberação de componentes (amido e proteínas), aumento da solubilidade do amido e da amilose (SIRISOONTARALAK e NOOMHORM, 2006). 
TABELA 2 - PROPRIEDADES DE COCÇÃO

\begin{tabular}{ccccc}
\hline \multirow{2}{*}{ PARÂMETROS } & \multicolumn{4}{c}{ AMOSTRAS } \\
\cline { 2 - 5 } & Controle & Dose 1 kGy & Dose 2 kGy & Dose 5 kGy \\
\hline Absorção de água (\%) & $347,4 \pm 6,8^{\mathrm{a}}$ & $337,4 \pm 2,1^{\mathrm{ab}}$ & $331,3 \pm 1,5^{\mathrm{b}}$ & $315,1 \pm 9,1^{\mathrm{c}}$ \\
Volume de expansão (\%) & $467,8 \pm 13,7^{\mathrm{a}}$ & $446,5 \pm 7,1^{\mathrm{ab}}$ & $432,2 \pm 21,5^{\mathrm{b}}$ & $435,7 \pm 27,3^{\mathrm{b}}$ \\
Perda de sólidos (\%) & $2,0 \pm 0,1^{\mathrm{b}}$ & $2,9 \pm 0,7^{\mathrm{b}}$ & $3,0 \pm 0,4^{\mathrm{b}}$ & $6,5 \pm 0,4^{\mathrm{a}}$ \\
Turbidez (T) & $89,1 \pm 1,1^{\mathrm{b}}$ & $88,8 \pm 1,2^{\mathrm{b}}$ & $91,4 \pm 0,5^{\mathrm{a}}$ & $92,2 \pm 0,3^{\mathrm{a}}$ \\
\hline
\end{tabular}

Valores reportados como média \pm desvio padrão. Médias seguidas pela mesma letra na linha não diferem entre si pelo Teste de Tukey $(p<0,05) . T$ = transmitância.

A turbidez da água de cocção do arroz foi maior nas doses mais elevadas de radiação, provavelmente devido às moléculas menores presentes nas amostras que receberam mais radiação. Assim, mesmo com mais sólidos em suspensão a transmitância foi maior, o que significa água menos opaca.

A análise de cor instrumental (Tabela 3) revelou que a luminosidade (L) das amostras não apresentou diferença estatística $(p<0,05)$, exceto entre as doses 2 e 5 kGy no arroz cru. Para o parâmetro Croma, observou-se que quanto maior a dose de radiação, maior a intensidade da cor tanto para o arroz cru quanto para o arroz cozido. O ângulo Hue apresentou tendência a se aproximar da cor amarela $\left(90^{\circ}\right)$ de forma mais intensa nas amostras cruas. O cozimento promoveu aumento do ângulo e diminuição da coloração amarela.

Zanão et al. (2009) observaram valores de L (67,9 a 70,3), C (10,0 a 14,1) e h $(95,1$ a 96,5$)$ para arroz cru semelhantes aos do presente estudo e coloração mais amarelada nas doses maiores (de 3 e 5 kGy), o que foi confirmado por Sirisoontaralak e Noomhorm (2006) que avaliaram arroz cru irradiado.

TABELA 3 - PARÂMETROS DE COR INSTRUMENTAL

\begin{tabular}{ccccc}
\hline PARÂMETROS & Controle & Dose 1 kGy & Dose 2 kGy & Dose 5 kGy \\
\hline \multicolumn{5}{c}{ ARROZ CRU } \\
\hline L & $69,15 \pm 0,91^{\mathrm{ab}}$ & $67,83 \pm 1,06^{\mathrm{ab}}$ & $69,37 \pm 1,19^{\mathrm{a}}$ & $67,60 \pm 0,37^{\mathrm{b}}$ \\
$\mathrm{h}$ & $10,26 \pm 0,59^{\mathrm{c}}$ & $10,71 \pm 0,62^{\mathrm{c}}$ & $13,08 \pm 0,72^{\mathrm{b}}$ & $15,07 \pm 0,71^{\mathrm{a}}$ \\
\hline $\mathrm{C}$ & $93,49 \pm 0,45^{\mathrm{ab}}$ & $93,26 \pm 0,37^{\mathrm{b}}$ & $94,42 \pm 0,59^{\mathrm{a}}$ & $94,38 \pm 0,44^{\mathrm{a}}$ \\
\hline $\mathrm{C}$ & ARROZ COZIDO & \\
\hline $\mathrm{C}$ & $72,76 \pm 2,30^{\mathrm{a}}$ & $72,87 \pm 2,47^{\mathrm{a}}$ & $73,53 \pm 0,89^{\mathrm{a}}$ & $72,03 \pm 3,86^{\mathrm{a}}$ \\
$\mathrm{h}$ & $3,82 \pm 0,55^{\mathrm{b}}$ & $4,37 \pm 0,53^{\mathrm{b}}$ & $5,17 \pm 0,90^{\mathrm{b}}$ & $7,68 \pm 0,49^{\mathrm{a}}$
\end{tabular}

Valores reportados como média \pm desvio padrão. Médias seguidas pela mesma letra na linha não diferem entre si pelo Teste de Tukey $(p<0,05)$.

\subsection{ANÁLISES MICROBIOLÓGICAS}

A pesquisa de microrganismos contaminantes do arroz revelou contagens inferiores a $1 \times 10^{-1}$ Unidades Formadoras de Colônias por grama de alimento (UFC/g) para coliformes totais e termotolerantes, Bacillus cereus e Estafilococos coagulase positiva e ausência de Salmonella 
sp. em $25 \mathrm{~g}$, em todas as amostras (0, 1, 2 e $5 \mathrm{kGy})$. Esses resultados permitiram o uso do arroz submetido à irradiação nos testes sensoriais para os julgadores sem oferecer risco à saúde.

Vale ressaltar que todas as amostras apresentaram valores abaixo do limite preconizado pela legislação brasileira, que tolera contagens até $1 \times 10^{1} \mathrm{UFC} / \mathrm{g}$ para coliformes a $45^{\circ} \mathrm{C}$, inferiores a $5 \times 10^{2} \mathrm{UFC/g}$ para Bacillus cereus e Estafilococos coagulase positiva e ausência de Salmonella sp. em $25 \mathrm{~g}$ para alimentos à base de cereais prontos para consumo (BRASIL, 2001).

\subsection{ANÁLISE SENSORIAL}

No teste de aceitação (Tabela 4), a maioria das amostras recebeu avaliação entre "gostei moderadamente" (nota 7) e "gostei muito" (nota 8) para todos os parâmetros avaliados. Diferença estatística $(p<0,05)$ foi observada apenas na dose de $5 \mathrm{kGy}$ para os parâmetros aroma e cor, que se enquadraram na escala de aceitação entre "gostei levemente" (nota 6) e "gostei moderadamente" (nota 7). Mediante esses resultados, nota-se que todas as doses de radiação aplicadas no arroz apresentaram boa aceitabilidade pelos julgadores.

TABELA 4 - TESTE DE ACEITAÇÃO SENSORIAL

\begin{tabular}{ccccc}
\hline \multirow{2}{*}{ PARÂMETROS } & \multicolumn{4}{c}{ AMOSTRAS } \\
\cline { 2 - 5 } & Controle & Dose $1 \mathrm{kGy}$ & Dose $2 \mathrm{kGy}$ & Dose $5 \mathrm{kGy}$ \\
\hline Aparência & $8,0 \pm 1,3^{\mathrm{a}}$ & $8,2 \pm 0,8^{\mathrm{a}}$ & $8,1 \pm 1,0^{\mathrm{a}}$ & $7,7 \pm 1,2^{\mathrm{a}}$ \\
Aroma & $7,4 \pm 1,3^{\mathrm{a}}$ & $7,3 \pm 1,4^{\mathrm{a}}$ & $7,2 \pm 1,5^{\mathrm{a}}$ & $6,6 \pm 1,5^{\mathrm{b}}$ \\
Sabor & $7,3 \pm 1,6^{\mathrm{a}}$ & $7,2 \pm 1,2^{\mathrm{a}}$ & $6,9 \pm 1,6^{\mathrm{a}}$ & $7,2 \pm 1,4^{\mathrm{a}}$ \\
Textura & $7,4 \pm 1,6^{\mathrm{a}}$ & $7,2 \pm 1,6^{\mathrm{a}}$ & $7,2 \pm 1,6^{\mathrm{a}}$ & $7,5 \pm 1,4^{\mathrm{a}}$ \\
Cor & $8,3 \pm 1,0^{\mathrm{a}}$ & $8,2 \pm 1,2^{\mathrm{a}}$ & $7,8 \pm 1,1^{\mathrm{a}}$ & $6,4 \pm 1,6^{\mathrm{b}}$ \\
\hline
\end{tabular}

Valores reportados como média \pm desvio padrão. Médias seguidas pela mesma letra na linha não diferem entre si pelo Teste de Tukey $(p<0,05)$.

Na Figura 1 estão dispostos os histogramas de distribuição das notas. Na dose de 1 kGy, $40 \%$ dos julgadores atribuíram nota máxima ( 9 = gostei muitíssimo) para o parâmetro aparência, e $42,5 \%$ nota 8 (gostei muito). A nota 9 foi atribuída por $17,5 \%$ dos julgadores para aroma, $15 \%$ para sabor, 22,5\% para textura e 52,5\% para cor. A nota 8 alcançou percentuais de $35 ; 22,5 ; 27,5$ e $25 \%$, respectivamente, para os mesmos parâmetros. As demais doses receberam percentuais favoráveis, entretanto, a dose de $1 \mathrm{kGy}$ mostrou melhor aceitação pelos julgadores.

Segundo Polesi et al. (2011), a dose de $1 \mathrm{kGy}$ foi benéfica para reduzir a digestibilidade do amido, com aumento do teor de amido resistente (arroz com teor calórico reduzido). Além disso, Zanão et al. (2009) observaram que essa dose assegurou resistência contra o ataque de insetos promovendo aumento da vida útil do arroz polido.

O coeficiente de correlação de Pearson para as variáveis cor sensorial e parâmetro $L$ no arroz cozido $(0,68)$ indica correlação positiva e moderada, ou seja, a diferença de luminosidade entre as amostras foi percebida pelos julgadores de forma mediana. Já o coeficiente entre a cor sensorial e os parâmetros $\mathrm{C}$ e h no arroz cozido (-0,99 e 0,98, respectivamente) evidencia forte correlação negativa com o croma e positiva com o ângulo Hue. Isso comprova que a cor modificada pela irradiação foi percebida pelos julgadores e detectada pelo colorímetro, sendo que quanto mais vívida a cor e mais próxima do amarelo menor foi a aceitabilidade por parte dos julgadores. 

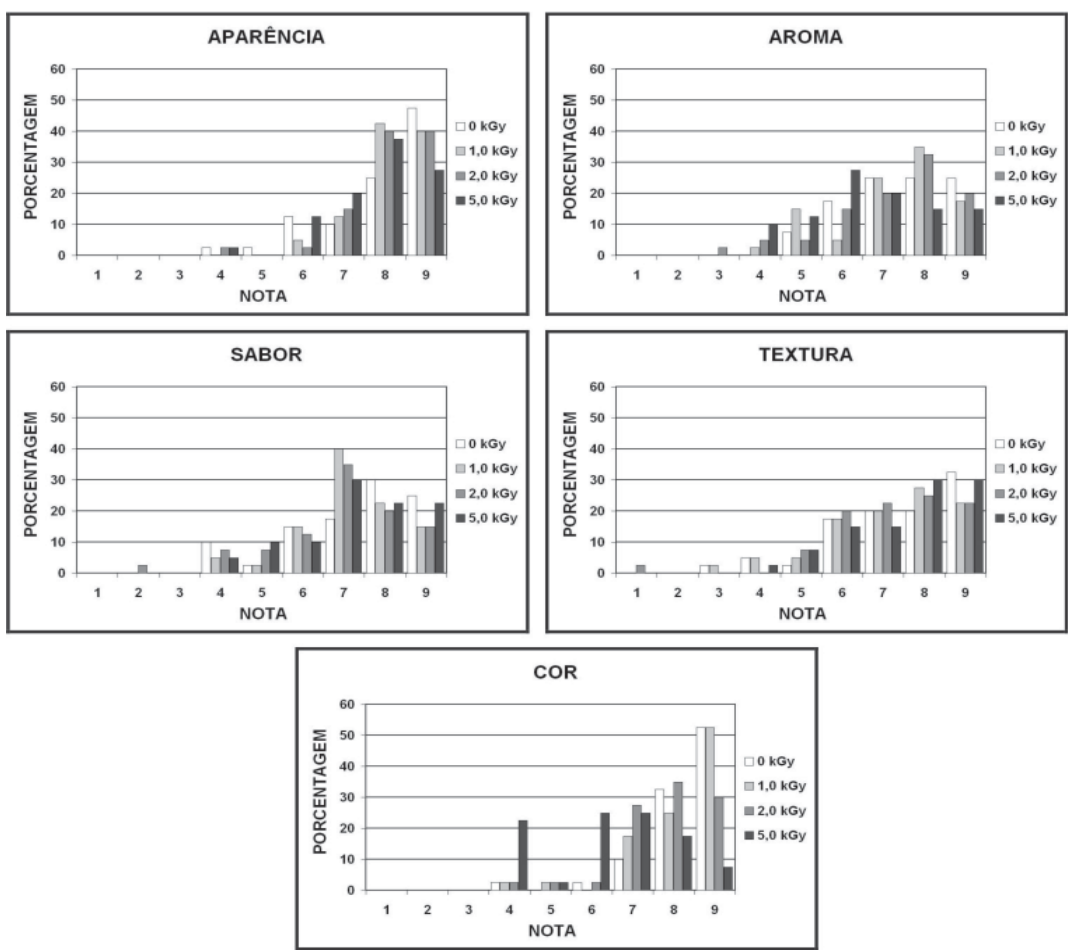

\section{FIGURA 1 - HISTOGRAMA DE DISTRIBUIÇÃO DE FREQUÊNCIA DE NOTAS DOS JULGADORES}

\section{CONCLUSÃO}

A radiação gama alterou as propriedades físico-químicas do arroz, com redução do teor de amido, da absorção deágua e do volume de expansão e aumento na perda de sólidos naágua de cocção e coloração amarela. Embora a irradiação tenha alterado essas propriedades, o arroz apresentou boa aceitabilidade pelos julgadores, exceto na dose de $5 \mathrm{kGy}$ para os parâmetros aroma e cor. A dose de radiação gama de 1 kGy não apresentou alteração em nenhum dos parâmetros avaliados, podendo ser aplicada em arroz polido mantendo suas características físico-químicas e aceitação pelo consumidor.

\section{ABSTRACT}

\section{IRRADIATION OF RICE AND CHANGES IN THEIR PHYSICOCHEMICAL AND SENSORY PROPERTIES}

The objective of this study was to evaluate the physicochemical properties and sensory acceptance of rice submitted to gamma radiation. Samples of commercial rice (IRGA 417), except for those on control group, were submitted to gamma radiation doses of 1,2 and $5 \mathrm{kGy}$, in dose rate of $0.5 \mathrm{kGy} / \mathrm{h}$. Irradiation promoted reduction in starch content and yellowing of grains. Regarding cooking properties, the rice presented lower water absorption and volume expansion, and greater loss of solids in cooking water as the radiation doses increased. Sensory evaluation showed good acceptance of rice by the panelists, except for the dose of $5 \mathrm{kGy}$. The $1 \mathrm{kGy}$ dose stood out as the best for irradiation of rice, because did not differ of the control in any parameter studied.

KEY-WORDS: GAMMA RADIATION; Oryza sativa; CENTESIMAL COMPOSITION; STARCH; COOKING PROPERTIES.

\section{REFERÊNCIAS}

1 AMERICAN PUBLIC HEALTH ASSOCIATION (APHA). Compendium of methods for the microbiological examination of foods. $4^{\text {th }}$ ed. Washington DC, 2001.

2 ASSOCIATION OF OFFICIAL ANALYTICAL CHEMISTRY (AOAC). Official methods of analysis of the AOAC International. $18^{\text {th }}$ ed. Arlington, 2005. $1141 \mathrm{p}$. 
3 BAO, J.; AO, Z.; JANE, J. L. Characterization of physical properties of flour and starch obtained from gamma-irradiated white rice. Starch/Stärke, v. 57, p. 480-487, 2005.

4 BASSINELLO, P. Z.; ROCHA, M. da S.; COBUCCI, R. de M. A. Avaliação de diferentes métodos de cocção de arroz de terras altas para teste sensorial. Santo Antônio de Goiás: Embrapa Arroz e Feijão, 2004. 8 p. (Embrapa Arroz e Feijão. Comunicado Técnico, 84).

5 BHAT, R.; KARIM, A. A. Impact of radiation processing on starch. Comprehensive Reviews in Food Science and Food Safety, v. 8, p. 44-58, 2009.

6 BRASIL. Agência Nacional de Vigilância Sanitária. RDC n 12, de 02 de janeiro de 2001. Regulamento técnico sobre padrões microbiológicos para alimentos. Diário Oficial [da] República Federativa do Brasil, Brasília, n.22-D, p.53-54, 10 jan. 2001.

7 BRASIL. Ministério da Agricultura, Pecuária e Abastecimento. Instrução Normativa nº 6, de 19 de fevereiro de 2009. Anexo I - Regulamento técnico do arroz. Diário Oficial [da] República Federativa do Brasil, Brasília, p. 7, 17 fev. 2009.

8 CHAVES, J. B. P.; SPROESSER, R. L. Práticas de laboratório de análise sensorial de alimentos e bebidas. Viçosa: UFV, 1996. $81 \mathrm{p}$.

9 CHUNG, H. J.; LIU, Q. Effect of gamma irradiation on molecular structure and physicochemical properties of corn starch. Journal of Food Science, v. 74, n. 5, p. C353-C361, 2009.

10 CUI, L.; PAN, Z.; YUE, T.; ATUNGULU, G.G.; BERRIOS, J. Effect of ultrasonic treatment of brown rice at different temperatures on cooking properties and quality. Cereal Chemistry, v. 87, p. 403-408, 2010.

11 DUTCOSKY, S.D. Análise sensorial de alimentos. Curitiba: Champagnat, 1996. 123 p.

12 FARKAS, J. Irradiation for better foods. Trends in Food Science and Technology, v.17, p.148-152, 2006.

13 FERREIRA, M .C.; PINHEIRO, B. da S.; SOUZA, I. S. F. de; MORAIS, O. P. de. Qualidade do arroz no Brasil: evolução e padronização. Santo Antônio de Goiás : Embrapa Arroz e Feijão, 2005. 61 p.

14 INSTITUTO BRASILEIRO DE GEOGRAFIA E ESTATÍSTICA (IBGE); MINISTÉRIO DA SAÚDE; MINISTÉRIO DO PLANEJAMENTO, ORÇAMENTO E GESTÃO. Pesquisa de orçamentos familiares 2008-2009. Análise do consumo alimentar pessoal no Brasil. Rio de Janeiro, 2011. 150 p.

15 LOZANO, J. E. El arte de evaluar los sabores. La Alimentación Latinoamericana, v. 33, n. 229, p. 42-33, 1999.

16 MINOLTA Co. Precise color communication: color control from perception to instrumentation. Osaka, 2007. 59 p.

17 ONG, M.H.; BLANSHARD, J.M.V. Texture determinants of cooked, parboiled rice. II. Physicochemical properties and leaching behaviour of rice. Journal of Cereal Science, v. 21, p. 261-269, 1995.

18 POLESI, L.F.; SARMENTO, S.B.S.; ARTHUR, V.; CANNIATTI-BRAZACA, S.G. Digestibilidade do amido e propriedades de cocção de arroz irradiado. In: SIMPÓSIO CIENTÍFICO DOS PÓS-GRADUANDOS DO CENA/USP, 4., 2011, Piracicaba. Anais.... Piracicaba: Centro de Energia Nuclear na Agricultura, 2011. p. 33.

19 RICKARD, J.E.; BEHN, K.R. Evaluation of acid and enzyme hydrolytic methods for the determination of cassava starch. Journal of the Science of Food and Agriculture, London, v. 41, n. 4, p. 373-379, 1987.

20 SIRISOONTARALAK, P.; NOOMHORM, A. Changes to physicochemical properties and aroma of irradiated rice. Journal of Stored Products Research, v. 42, p. 264-276, 2006.

21 SOMOGY, M. A new reagent for the determination of sugar. The Journal of Biological Chemistry, Baltimore, v. 160, n.1, p. $61-68,1945$

22 STATISTICAL ANALYSES SYSTEM (SAS). SAS/INSIGHT user's guide. V. 9.1.3 for windows. Cary, 2002/2003.

23 UNIVERSIDADE DE CAMPINAS (UNICAMP). Tabela brasileira de composição de alimentos (TACO). Campinas: NEPA-UNICAMP, 2006. $113 \mathrm{p}$

24 UNIVERSIDADE DE SÃO PAULO (USP). Tabela brasileira de composição de alimentos (TBCA-USP 5.0). Disponível em: < http://www.fcf.usp.br/tabela/resultado.asp?IDLetter=A\&IDNumber=208>. Acesso em: 11 out. 2011.

25 WALTER, M.; MARCHEZAN, E.; AVILA, L. A. Arroz: composição e características nutricionais. Ciência Rural, v. 38, n. 4, p.1184-1192, 2008.

26 WOOTTON, M.; DJOJONEGORO, H.; DRISCOLL, R. The effect of $\gamma$-irradiation on the quality of Australian rice. Journal of Cereal Science, v. 7, p. 309-315, 1988.

27 ZANÃO, C.F.P.; CANNIATTI-BRAZACA, S.G.; SARMENTO, S.B.S.; ARTHUR, V. Efeito da irradiação gama nas características físico-químicas e sensoriais do arroz (Oryza sativa L.) e no desenvolvimento de Sitophilus oryzae L. Ciência e Tecnologia de Alimentos, v. 29, p. 46-55, 2009. 